\title{
Paired Gap States in a Semiconducting Carbon Nanotube: Deep and Shallow Levels
}

\author{
Sungjun Lee, ${ }^{1,2}$ Gunn Kim, ${ }^{1}$ Hajin Kim, ${ }^{1,2}$ Byoung-Young Choi, ${ }^{1,2}$ Jhinhwan Lee, ${ }^{1,2}$ Byoung Wook Jeong, ${ }^{1}$ Jisoon Ihm, ${ }^{1}$ \\ Young Kuk, ${ }^{1,2}$ and Se-Jong Kahng ${ }^{3}$ \\ ${ }^{1}$ School of Physics, Seoul National University, Seoul, 151-742, Korea \\ ${ }^{2}$ Center for Science in Nanometer Scale, Seoul National University, Seoul, 151-742, Korea \\ ${ }^{3}$ Department of Physics, Korea University, Seoul, 136-713, Korea
}

(Received 22 March 2005; published 12 October 2005)

\begin{abstract}
Several paired, localized gap states were observed in semiconducting single-wall carbon nanotubes using spatially resolved scanning tunneling spectroscopy. A pair of gap states is found far from the band edges, forming deep levels, while the other pair is located near the band edges, forming shallow levels. With the help of a first-principles study, the former is explained by a vacancy-adatom complex while the latter is explained by a pentagon-heptagon structure. Our experimental observation indicates that the presence of the gap states provides a means to perform local band-gap engineering as well as doping without impurity substitution.
\end{abstract}

DOI: 10.1103/PhysRevLett.95.166402

For the development of future electronic devices, carbon nanotubes (CNTs) have been considered as model onedimensional systems. Various unit electronic devices including logic gates have been demonstrated using CNTs $[1,2]$. It was suggested that the device characteristics could be controlled by functionalized CNTs with inserted molecules, atomic defects, and chemical dopants [3-6]. With inserted molecules, for example, the band gaps of semiconducting CNTs can be locally engineered. Inherent atomic defects are often found at intramolecular junctions, joining two different CNTs. The junction defects were studied and understood in analogy to conventional bulkbulk interfaces [7-10]. Without junction structures, however, atomic defects are often present on the wall of a single CNT [11-20]. Such simple atomic defects are much more abundant than junction defects in as-grown CNTs. It was reported, on the basis of transmission electron microscopy, that simple atomic defects, created by electron irradiation, may initiate diameter reduction of CNTs, followed by breaking of the whole structure $[11,12]$. It was suggested that the simple atomic defects can play a crucial role in the mechanical ductility and electrical resistivity of CNTs [15-19]. Atomic defects in CNTs may be classified into two different types. One is the Stone-Wales type whose basic unit is a pentagon-heptagon structure. Here, the network topology deviates from the six-membered hexagon structure. The other is the vacancy-adatom type whose basic unit is a vacancy and/or an adatom on the CNT wall, resulting in a local deficiency or excess of carbon atoms. There have been extensive theoretical studies for these two types of atomic defect [11-20], but no quantitative analysis has been reported in comparison with actual experimental observations so far.

In this Letter, we report on the electronic local density of states (LDOS) of a semiconducting CNT, measured with a low-temperature scanning tunneling microscope (STM). Several paired, localized gap states are studied with spa-
PACS numbers: 71.20.Tx, 61.48.+c, 68.37.Ef, 81.07.De

tially resolved scanning tunneling spectroscopy (STS), and two pairs are analyzed and reported here. One pair of gap states is explained by a vacancy-adatom complex, while the other pair is explained by a pentagon-heptagon structure. The latter states merge with the valence and conduction band edges, exhibiting a narrowing of band gap of a semiconducting CNT.

Single-wall CNTs were sonicated for $>1 \mathrm{~h}$ in dichloroethane to produce a uniform colloidal suspension. An $\mathrm{Au}(111)$ substrate was cleaned by repeated cycles of sputtering and annealing in ultrahigh vacuum (UHV). After that, a droplet of the CNT suspension was dispersed onto the $\mathrm{Au}(111)$ substrate with a glass pipette. To avoid contamination in the ambient environment, we performed this procedure in a glove box, filled with pure Ar gas, connected to the UHV chamber through a load-lock system. When the solvent in the droplet was completely dried on the $\mathrm{Au}(111)$ substrate, the sample was put back into the UHV chamber with a STM. In the UHV chamber, the sample was heated again up to $\sim 150{ }^{\circ} \mathrm{C}$ to remove any residual solvent. We observed isolated and bundled CNTs. Well-known herringbone reconstructions were observed on some parts of the sample, even after these consecutive UHV-Ar environment-UHV procedures. All the measurements were done with our home-built low-temperature STM [21], which can be operated at 4.7 or $78 \mathrm{~K}$.

Figure 1(a) shows a STM image and Fig. 1(b) shows a close-up view of a derivative STM image of a CNT. The STS data (LDOS map) of the CNT is shown in Fig. 1(c). Atomic structures are clearly resolved in the derivative STM image. On the basis of the LDOS map in Fig. 1(c), which will be explained shortly, the CNT can be divided into four regions labeled as A, B, C, and D. The atomic patterns of regions $\mathrm{B}$ and $\mathrm{C}$ are dissimilar to those of regions $\mathrm{A}$ and $\mathrm{D}$ in Fig. 1(a), implying that both topographical and/or electronic structures are different in these two regions. The value of $d I / d V$ is approximately propor- 
(a)

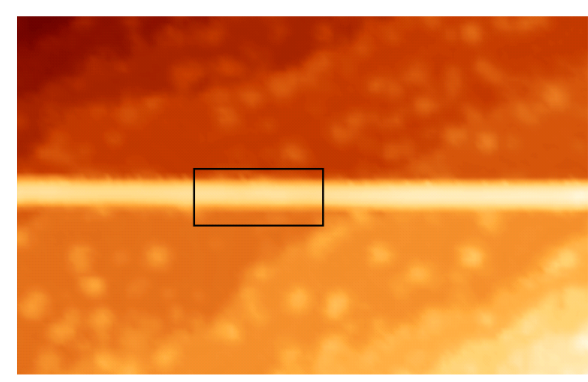

(b)
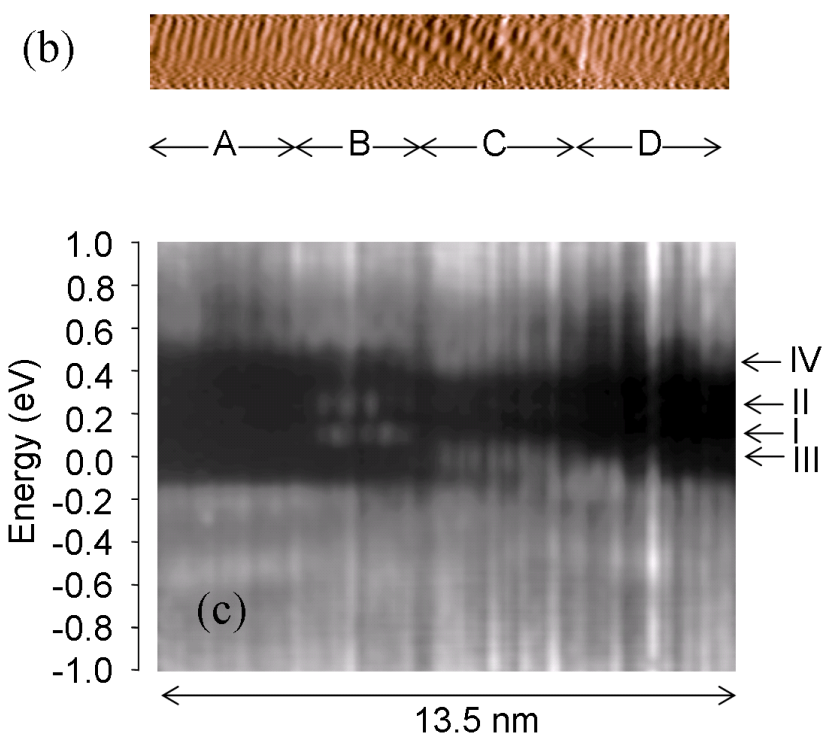

FIG. 1 (color online). (a) A STM image with a CNT. The image size is $60 \times 39 \mathrm{~nm}$. (b) A derivative STM image. This is zoomed from the rectangle area of (a). The image length is $13.5 \mathrm{~nm}$. (c) The spatially resolved LDOS map (STS data) for the CNT (a). On the basis of the STS data, the CNT is divided into regions $\mathrm{A}, \mathrm{B}, \mathrm{C}$, and $\mathrm{D}$, respectively. The labels I, II, III, and IV are the energy levels for gap states.

tional to the LDOS [22]. In this experiment, the map of $d I /$ $d V$ is obtained by the lock-in technique, as a function of both the position along the CNT axis and the sample bias voltage. The fixed tunneling gap for $d I / d V$ was set by the tunneling current during the sampling time period, in sample-and-hold mode. The result is presented as a twodimensional image, in which brightness is proportional to the LDOS, as shown in Fig. 1(c). Figure 2 shows four LDOS spectra sampled from regions A to D. In practice, they are the selections of vertical line profiles from Fig. 1(c).

In Fig. 1(c), region A shows the typical shape of an LDOS map for semiconducting CNTs. The valence band maximum (VBM) is located at $-0.08 \mathrm{eV}$, and the conduction band minimum (CBM) is located at $0.46 \mathrm{eV}$. Between the VBM and the CBM, the LDOS is absent in region A, as shown in Fig. 2 (spectrum A). Thus, the CNT is semiconducting with a band gap of $0.54 \mathrm{eV}$ and is $p$ type. CNTs are often observed to be $p$ type, which is understood

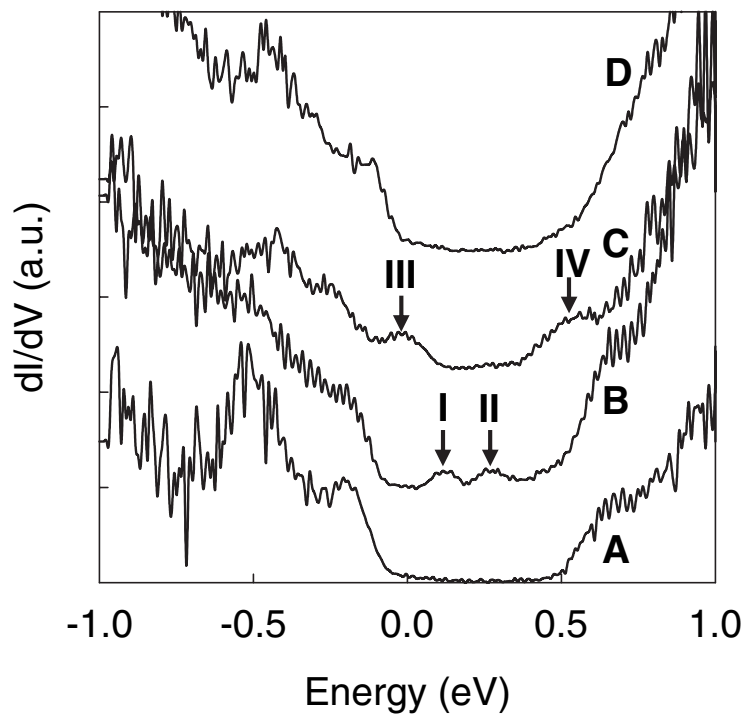

FIG. 2. Sampled STS data, from regions A to D of Fig. 1(c). The curves from B, C, and D are shifted for clearer presentation. The arrows indicate gap states.

in terms of charge donation to the substrate, due to the work function difference between an $\mathrm{Au}(111)$ substrate $(\sim 5.3 \mathrm{eV})$ and CNTs $(\sim 4.8 \mathrm{eV})$. The energy levels of both the $\mathrm{CBM}$ and the VBM are constant throughout regions $\mathrm{A}$ and $\mathrm{B}$. In region $\mathrm{B}$, there is an additional pair of localized states within the band gap. One is located at $0.12 \mathrm{eV}$, and the other is located at $0.24 \mathrm{eV}$, in Fig. 2 (spectrum B, the lower state labeled I, and the higher one II). Since they are almost at the center of the band gap and far from the band edges, they can be termed deep levels, following the naming convention for bulk semiconductors. In Fig. 3, the horizontal (or spatial) line profiles of the LDOS map are shown for these deep levels I and II. The spatial locations

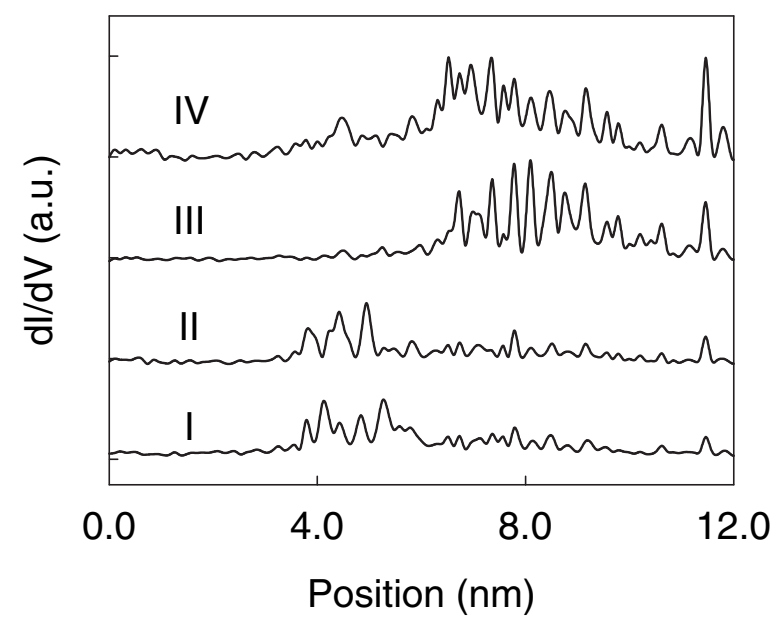

FIG. 3. Horizontal line profiles of Fig. 1(c). The profile I is at $0.12 \mathrm{eV}$, II is at $0.24 \mathrm{eV}$, III is at $0.02 \mathrm{eV}$, and IV is at $0.48 \mathrm{eV}$. The profiles of II, III, and IV are shifted upward for clearer presentation. 
of these deep levels are coincident, and the amplitude distributions resemble each other. The pair of deep levels originates from a single defect structure which will be discussed later. The decay lengths of the deep levels are estimated to be $\sim 2 \mathrm{~nm}$.

In Fig. 2, the spectrum $\mathrm{C}$ shows peaks at 0.02 and $0.48 \mathrm{eV}$ (the lower state labeled III and the higher one IV). The apparent band gap in region $\mathrm{C}$ is smaller than that in region A (and B), as shown in Fig. 1(c). It was predicted by the first-principles theory that a pentagon-heptagon structure induces shallow levels (again following the naming convention for bulk semiconductors) and that they can be resonating or merging with band edges $[4,10]$. When the gap states in the shallow level merge with band edges, the band gap may look locally narrowed. In Fig. 3, the horizontal (or spatial) line profiles of the LDOS map are shown for these two states. Both of them are localized in region C with a decay length of $\sim 3 \mathrm{~nm}$. Since their amplitudes are almost the same and also their spatial distributions are comparable to the pentagon-heptagon defect states in Ref. [10], we conjecture that these localized states are caused by a pentagon-heptagon structure. According to Hückel's rule [23], a hexagon (with $6 \pi$ electrons) is more stable than a pentagon (with $5 \pi$ electrons) or a heptagon (with $7 \pi$ electrons). Thus, a heptagon gives up an electron to its neighbors and produces a donor level in semiconducting CNTs. Similarly, a pentagon produces an acceptor level. The observed lower energy state at $0.02 \mathrm{eV}$, therefore, is assigned to be a shallow acceptor level produced by a pentagon, while higher energy state at $0.48 \mathrm{eV}$ is a shallow donor level produced by a heptagon. The pentagon-heptagon structure producing the pair of gap states, is unfortunately not well resolved in our STM images, probably because they are located at a side or bottom wall of the CNT, which the STM tip cannot access.

In order to understand the observed spectra in more detail, we have performed a first-principles study, based on the density functional theory [24] with a local density approximation [25] for the exchange-correlation term. We have used the SIESTA code [26]. The ionic potential is described by the norm-conserving Troullier-Martins pseudopotential [27]. We expand the wave functions in a double-zeta basis set with an energy cutoff (for real space mesh points) of $80 \mathrm{Ry}$. We choose a $(17,0)$ zigzag CNT of $\sim 13.4 \AA$ in diameter. The supercell in the lateral direction is larger than $25 \AA$ and it has 5 unit cells in the tube axis direction.

At first, to understand deep levels, we tested many different vacancy-adatom geometries. Vacancies alone do not reproduce the experimental spectra of two deep levels. When we introduce a vacancy-adatom complex (a vacancy plus $1 \mathrm{C}$ atom outside the wall), as illustrated in Fig. 4(a), two unoccupied flat levels are produced in the semiconducting gap region, as plotted in Fig. 4(b). The energy spacing between the two flat bands is $0.13 \mathrm{eV}$, in agreement (a)
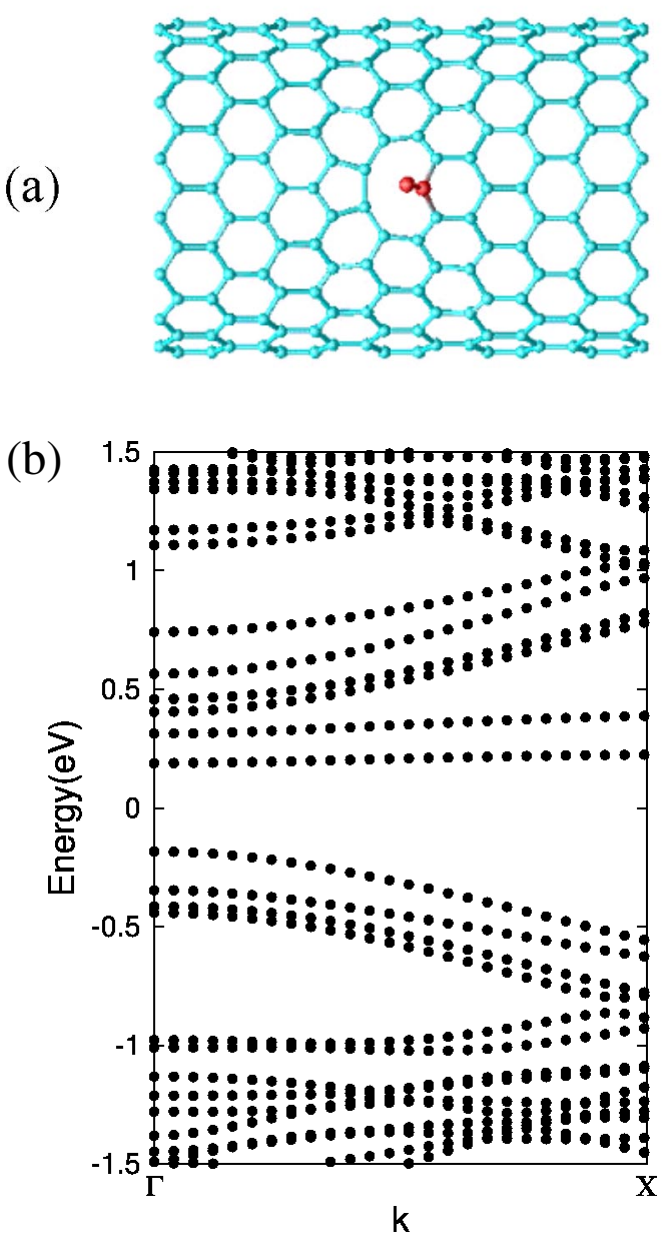

FIG. 4 (color online). Atomic model and band structure of semiconducting CNTs with defects. (a) Atomic model of a vacancy-adatom complex on the $(17,0) \mathrm{CNT}$, (b) band structure corresponding to the $(17,0)$ CNT with the vacancy-adatom complex in (a).

with experimental data in region B in Figs. 1(c) and 2. The calculated energy position of the gap states is slightly higher than experiment. In the relaxed geometry of the vacancy-adatom complex, two of three carbon atoms around the vacancy rebond and the adatom bonds to the third atom to saturate the dangling bond. Our results have similar features to other theoretical works [20]. Previously we and anther group performed extensive calculations $[10,28]$ for various pentagon-heptagon defect structures and obtained two shallow levels, one acceptorlike and the other donorlike, which we will not present here again. Although our model in Fig. 4 is not the only one to reproduce experimental observations, it is one of the simplest local defects which show the major features of the experiment.

The magnitude of the imaginary wave vector (inverse of the decay length) in the band gap is proportional to $\left|E_{c(V)}-E_{d}\right|^{1 / 2}$ where $E_{c(V)}$ is the energy of the CBM (VBM) and $E_{d}$ is the energy of the defect state. As ex- 
pected from this, Fig. 3 shows that the shallow level extends more than the deep level. The lifetime of electrons in a localized state can be estimated with the linewidth of the LDOS spectrum. The linewidths of the deep levels are measured to be $\sim 0.1 \mathrm{eV}$. They are determined by the instrumental broadening and a value proportional to the inverse lifetime. Since the linewidths of the shallow levels are $\sim 50 \%$ larger than those of deep levels, the lifetimes of shallow levels may be shorter than those of deep levels. Finally, in region D, there is a parallel upward shift of conduction and valence band edges. This parallel shift may occur when this local area loses more charge to the substrate, possibly due to a better contact, than the other parts of CNT.

In summary, several paired gap states were observed in a semiconducting single-wall carbon nanotube with spatially resolved scanning tunneling spectroscopy. A pair of gap states, forming deep levels, is caused by a vacancy-adatom complex. The other pair of gap states, manifesting itself in the merged shoulder structure of band edges in LDOS spectra, is analyzed and assigned as having shallow acceptor and donor levels. The shallow levels are explained by a pentagon-heptagon structure. This experimental observation confirms a recent theoretical prediction that induced gap states can work as a means to achieve doping and local band-gap engineering as well as doping without impurity substitution.

We acknowledge financial support by the Ministry of Science and Technology of Korea (National Creative Research Initiatives, CNNC of Sungkyunkwan University, and M10503000187), by the Ministry of Commerce, Industry and Energy of Korea (National R\&D Project for Nano-Science and Technology), and by Korea Research Foundation (KRF-2003-015-C00208).

[1] M.S. Dresselhaus, G. Dresselhaus, and Ph. Avouris, Carbon Nanotubes: Synthesis, Structures, Properties, and Application (Springer-Verlag, New York, 2001).

[2] C. Dekker, Phys. Today 52, No. 5, 22 (1999).

[3] J. Lee et al., Nature (London) 415, 1005 (2002).

[4] Y. Cho, S. Han, G. Kim, H. Lee, and J. Ihm, Phys. Rev. Lett. 90, 106402 (2003).
[5] Z. Yao, H.W. Ch. Postma, L. Balents, and C. Dekker, Nature (London) 402, 273 (1999).

[6] C. Zhou, J. Kong, E. Yenilmez, and H. Dai, Science 290, 1552 (2000).

[7] M. Ouyang, J.-L. Huang, C. L. Cheung, and C. M. Lieber, Science 291, 97 (2001).

[8] L. C. Venema, J. W. Janssen, M. R. Buitelaar, J.W. G. Wildoer, S.G. Lemay, L.P. Kouwenhoven, and C. Dekker, Phys. Rev. B 62, 5238 (2000).

[9] M. Ishigami, H. J. Choi, S. Aloni, S. G. Louie, M. L. Cohen, and A. Zettle, Phys. Rev. Lett. 93, 196803 (2004).

[10] H. Kim et al., Phys. Rev. Lett. 90, 216107 (2003).

[11] J.-C. Charlier, T. W. Ebbesen, and Ph. Lambin, Phys. Rev. B 53, 11108 (1996).

[12] P. M. Ajayan, V. Ravikumar, and J.-C. Charlier, Phys. Rev. Lett. 81, 1437 (1998).

[13] V. H. Crespi, N. G. Chopra, M. L. Cohen, A. Zettle, and S. G. Louie, Phys. Rev. B 54, 5927 (1996).

[14] V. H. Crespi, M. L. Cohen, and A. Rubio, Phys. Rev. Lett. 79, 2093 (1997).

[15] V. Meunier, P. Senet, and Ph. Lambin, Phys. Rev. B 60, 7792 (1999)

[16] D. Orlikowski, M. B. Nardelli, J. Bernholc, and C. Roland, Phys. Rev. B 61, 14194 (2000).

[17] A. Hansson, M. Paulsson, and S. Stafstrom, Phys. Rev. B 62, 7639 (2000).

[18] A. V. Krasheninikov and K. Nordlund, Surf. Sci. 454, 519 (2000).

[19] A. J. Lu and B.C. Pan, Phys. Rev. Lett. 92, 105504 (2004).

[20] J. Rossato, R. J. Baierle, A. Fazzio, and R. Mota, Nano Lett. 5, 197 (2005).

[21] J.-Y. Park, Ph.D. thesis, Seoul National University (2000).

[22] J. Tersoff and D. R. Hamann, Phys. Rev. B 31, 805 (1985).

[23] T. W. G. Solomons, Organic Chemistry (Wiley, New York, 1996), 6th ed., p. 624.

[24] W. Kohn and L. J. Sham, Phys. Rev. 140, A1133 (1965).

[25] D. M. Ceperley and B. J. Alder, Phys. Rev. Lett. 45, 566 (1980).

[26] P. Ordejón, E. Artacho, and J. M. Soler, Phys. Rev. B 53, R10441 (1996).

[27] N. Troullier and J. L. Martins, Phys. Rev. B 43, 1993 (1991).

[28] W. Fa, J. Chen, and J. Dong, Eur. Phys. J. B 37, 473 (2004). 